\title{
USO DE ATRAYENTES Y SUPLEMENTOS ALIMENTICIOS PARA EL INCREMENTO DE DEPREDADORES DE ESCAMA BLANCA DEL MANGO, AULACASPIS TUBERCULARIS NEWSTEAD (HEMIPTERA: DIASPIDIDAE)
}

\author{
Luis Martín HernándeZ-FUENTES, ${ }^{1}$ Mario Alfonso URÍAS-LÓPEZ, ${ }^{1}$ \\ JOSÉ ISABEL LÓPEZ ARROYO ${ }^{2}$ \& JAIME GUSTAVO LÓPEZ ARRIAGA ${ }^{\mathbf{1}}$ \\ ${ }^{1}$ Inifap. Campo Experimental Santiago Ixcuintla. Carret. Internacional México-Nogales, Km. 6 \\ Entronque a Santiago Ixcuintla. C.P. 63300. Santiago Ixcuintla, Nayarit. México. \\ ${ }^{2}$ Inifap. Campo Experimental General Teran km. 31 carretera Montemorelos-China, Col. Exhacienda \\ Las Anacuas. C.P. 67400. Nuevo León, México. \\ $<$ hernandez.luismartin@inifap.gob.mx>
}

Hernández-Fuentes, L. M., M. A. Urías-López, J. I. López Arroyo \& J. G. López Arriaga. 2012. Uso de atrayentes y suplementos alimenticios para el incremento de depredadores de escama blanca del mango, Aulacaspis tubercularis Newstead (Hemiptera: Diaspididae). Acta Zoológica Mexicana (n. s.), 28(1): 145-160.

RESUMEN. El mango es un cultivo importante en México, en años recientes se ha observado un incremento poblacional de Aulacaspos tubercularis en este frutal. Se evaluaron atrayentes y suplementos alimenticios para retener y/o atraer depredadores de $A$. tubercularis en dos ciclos de producción (20092010). Se observó mayor efecto de tratamientos en la variable huevos de Ceraeochrysa con el uso de $0.25 \mathrm{~mL}$ de salicilato de metilo (SM) por árbol. Adultos de Pentilia fueron ligeramente atraídos con las diferentes dosis de SM. Con el uso de $4 \mathrm{~mL}$ de SM por árbol, se observó $60 \%$ menos colonias de ninfas de A. tubercularis comparado con el testigo absoluto. En el primer año de evaluación no se observaron adultos de Chilochorus cacti, en cambio en el segundo año en los tratamientos con azúcar, levadura de cerveza y leche nido en polvo si se presentaron. Con la aplicación de suplementos alimenticios a base de agua + levadura de cerveza y azúcar, se observó una diferencia numérica importante de huevos de $\mathrm{Ce}$ raeochrysa sp. El número de colonias de A. tubercularis por hoja, claramente disminuyó en el segundo año de evaluación en ambos sitios, en el sitio uno la disminución de colonias en promedio fue de $65.6 \%$ y para el sitio dos fue de un $93 \%$. Esta disminución podría ser el resultado de un incremento general de los depredadores y la acción conjunta de estos sobre las colonias de escamas.

Palabras clave: Plagas, mango, atrayentes, control biológico.

Hernández-Fuentes, L. M., M. A. Urías-López, J. I. López Arroyo \& J. G. López Arriaga. 2012. Use of attractants and nutritional supplements to increase mango white scale's, Aulacaspis tubercularis Newstead (Hemiptera: Diaspididae) predators. Acta Zoológica Mexicana (n. s.), 28(1): 145-160.

Recibido: 11/05/2011; aceptado: 08/12/2011. 
ABSTRACT. Mango is an economically important crop in Mexico, important population increases of mango white scale Aulacaspis tubercularis have been observed. The application of attractive nutritional supplements to arrest and/or attract predators of A. tubercularis were evaluated during two seasons. Application of $0.25 \mathrm{~mL}$ of methyl salicilate per tree has an effect on the number of eggs of Ceraeochrysa spp. Adults of Pentilia spp were lured whit different doses of methy salicilate (MS). Whit $4 \mathrm{~mL}$ of MS per tree, $60 \%$ fewer groups of $A$. tubercularis nymphs occurred. In the first year, adults of the predator Chilochorus cacti were no observed, but in the second year, the use of sugar, yeast and powdered milk had an attractive effect. More eggs of Ceraeochrysa spp were observed when water plus yeast plus sugar treatments were applied. At two sites, nymphs of $A$. tubercularis decreased in the second year, in the first site decrease reached $65.6 \%$ while in the second site it was $93 \%$ in comparison to the untreated controls. The observed abatement is attributed to a general increase in predator numbers and their action on pest population.

Keys words: pests, mango, attractants, biological control.

\section{INTRODUCCIÓN}

El mango (Mangifera indica L.) es uno de los frutales tropicales más importantes a nivel mundial. México ocupa el cuarto lugar en producción después de Tailandia (FAOSTAT 2007). Se estima una superficie cultivada de 183,892 ha distribuidas en 23 estados del país, con un valor de la producción de 3.99 billones de pesos; Nayarit, ocupa el sexto lugar nacional con 21,746 ha y un valor de la producción de 479.79 millones de pesos (SIAP-SAGARPA 2009). En este estado, en los últimos 6 años se han observado diversos problemas fitosanitarios de importancia económica y cuarentenaria; entre éstos se encuentran la escama blanca del mango EBM (Aulacaspis tubercularis Newstead) y la cochinilla rosada del hibisco (Maconelliccocus hirsutus Green) (Urías 2006, Urías et al. 2010). La hembra de EBM es de forma ovalada, plana y de color blanco transparente, se localiza en el haz y envés de las hojas (Peña \& Mohyuddin 1997) y frutos (Urías \& Flores 2005), provoca daños por alimentarse de hojas y frutos, genera un manchado de estos últimos debido a la toxicidad de su saliva (Peña \& Mohyuddin 1997), en fuertes infestaciones induce caída del follaje y muerte de ramas (Wysoki et al. 1993); es capaz de provocar hasta $50 \%$ de pérdidas en la cosecha (Urías 2006).

Aunque la EBM puede ser controlada con diversos productos químicos, aceites y detergentes (García et al. 2009), no es recomendable su uso ya que algunos aceites pueden causar daños a las inflorescencias (observación personal-no publicado); la aplicación de insecticidas puede afectar a los enemigos naturales (Jepson 1989, Waage 1989, Croft 1990); para el control de escamas que atacan el mango, el uso de enemigos naturales ha sido un método eficaz (Galán 1999). Para EBM, existen diversos depredadores y parasitoides; en Sudáfrica Labuschagne (1993), consigna a Auleurodothrips fasciapennis Franklin y Aspidiotiphagus citrinus Craw como los más importantes; en Israel se reporta a Coccophagus lycimnia Walker, C. eritraensis Compere, C. scutellaris Dalman, C. bivittatus Compere, Microterys flavus Howard 
y Metaphicus flavus Howard como parasitoides de EBM y el control químico no es requerido debido a la acción de estos enemigos naturales (Kfir \& Rosen, 1980). En Nayarit, México, se han observado diversos depredadores, figuran entre ellos $\mathrm{Ce}$ raeochrysa sp., Leucochrysa sp, Chrysoperla externa Hagen, Chilocorus cacti L., Azya orbigera Mulsan y Pentilia sp. (Urías \& Flores 2005, González et al. 2010).

Los agentes de control biológico se pueden utilizar de diversas maneras para controlar a las plagas agrícolas: por conservación, introducción y por incremento (Stehr 1990); en la primera estrategia, los enemigos naturales deben ser suficientemente abundantes y atacar a las plagas de interés; existen diferentes prácticas para mejorar la actividad de los enemigos naturales, entre ellas, proporcionar alimento y fuentes de proteínas y carbohidratos cuando estos recursos son limitados (Van Driesche \& Bellows 1996). El uso de control biológico por conservación (CBC) como componente del manejo integrado de plagas es una estrategia que esta aumentando en importancia y popularidad (Barbosa 1998). Cardinale et al. (2003) señalaron que la investigación en $\mathrm{CBC}$ en muchos sistemas de cultivo se centra en fortalecer la comunidad de enemigos naturales, en términos de densidad de población y diversidad de especies. El CBC, como una estrategia que ayuda a los enemigos naturales especialistas y generalistas, es visto como una estrategia dentro del manejo integrado de plagas para mejorar la protección de cultivos (James et al. 2005). La intención del CBC mediante la aplicación de suplementos alimenticios y atrayentes es atraer y/o retener a los enemigos naturales en el cultivo y aumentar la relación depredador y parasitoide/presa para llevar a la población plaga a niveles que no causen un daño económico al cultivo (Jervis \& Heimpel, 2007).

Los insectos herbívoros diariamente están sometidos a olores y esencias naturales atrayentes, las cuales utilizan como señales de orientación para encontrar su fuente de alimento, sitio de apareamiento, sitio de oviposicion y como sistema de alarma (Dethier 1947). La planta, al ser atacada por insectos y ácaros libera sustancias químicas que atraen a depredadores, por ejemplo, el Salicilato de Metilo (SM) es comúnmente liberado por las plantas al ser estas infestadas y es a su vez un atrayente para depredadores (DeBoer \& Dicke 2004, citado por Lee 2010), tal es el caso de Phaseolus lunatus que al ser infestada por Tetranychus urticae Koch libera, entre otros compuestos, SM, los cuales atraen al depredador Phytoseiulus persimilis Athias-Henriot (Dicke et al. 1999). La emisión de SM por plantas dañadas por insectos y ácaros y el papel que este desempeña como atrayente de depredadores ha sido consignada en diferentes publicaciones (Dicke et al. 1990, 1999; James 2003; De Boer \& Dicke 2003; Zhu \&Park 2005; Hegde et al. 2011). Evaluaciones en campo sobre la aplicación práctica de SM para atraer y/o retener depredadores han sido poco realizadas. Al respecto, Lee (2010) menciona que en fresa se pueden prevenir altas infestaciones de pulgones y ácaros al aplicar SM ya que éste atrae una variedad de depredadores. Por su parte Zhu \& Park (2005) observaron atracción de Coccinella septempunctata L. en trampas 
cebadas con SM. El uso de los compuestos volátiles que libera la planta al ser estimulada por los herbívoros, representa una estrategia con gran potencial para el desarrollo de métodos efectivos y prácticos basados en el uso de semioquímicos para manipular las poblaciones de enemigos naturales. Comparado con los estudios realizados en laboratorio, las evaluaciones de atrayentes en campo para retener y/o incrementar los enemigos naturales, son aún poco numerosas (James et al. 2005).

El objetivo del presente estudio fue evaluar el efecto de suplementos alimenticios y salicilato de metilo para incrementar y/o retener insectos depredadores de escama blanca del mango Aulacaspis tubercularis Newstead.

\section{MATERIAL Y MÉTODO}

La investigación se realizó en dos huertos de mango var. Ataulfo, en dos ciclos de producción durante los años 2009 y 2010 en dos municipios del estado de Nayarit, México. Para diferenciar los lugares de evaluación, se identificaron como sitio uno y dos.

Sitio uno. Se ubicó en los terrenos del ejido El Capomo, Compostela, Nayarit (Latitud Norte $21^{\circ} 07^{\prime} 11^{\prime \prime}$ y Longitud Este $105^{\circ} 11^{\prime} 12.4^{\prime \prime}$. A $60 \mathrm{msnm}$ ), en un huerto de 12 años de edad, plantado en marco real a una distancia de $10 \times 10 \mathrm{~m}$. La humedad relativa y temperatura promedio durante los dos ciclos de evaluación fueron: $72.5 \%$ y $24.75{ }^{\circ} \mathrm{C}, 77.4 \%$ y $22.7^{\circ} \mathrm{C}$, en el primer y segundo ciclo, respectivamente. Los tratamientos se enlistan en el cuadro 1 .

Las aplicaciones en el primer ciclo iniciaron el 13 de marzo y concluyeron el 08 de mayo de 2009, en la segunda evaluación iniciaron el 5 de febrero y concluyeron el 15 de mayo de 2010. El salicilato de metilo (SM) se vertió en un liberador gradual constituido por un frasco de plástico transparente de $100 \mathrm{~mL}$ de capacidad y con cinco perforaciones de $1 \mathrm{~mm}$ de diámetro ubicadas a la mitad del frasco. El liberador se

Cuadro 1. Suplementos alimenticios y atrayente evaluados para atraer y/o retener depredadores de escama blanca del mango Aulacaspis tubercularis. Sitio uno. El Capomo, Nayarit. 2009-2010.

\begin{tabular}{cll}
\hline Tratamiento & Descripción & Dosis \\
\hline 1 & Testigo absoluto & Sin aplicación \\
2 & Salicilato de metilo & $0.25 \mathrm{~mL} /$ bote/árbol \\
3 & Salicilato de metilo & $0.5 \mathrm{~mL} / \mathrm{bote} /$ árbol \\
4 & Salicilato de metilo & $1 \mathrm{~mL} /$ bote/árbol \\
5 & Salicilato de metilo & $2 \mathrm{~mL} /$ bote/árbol \\
6 & Salicilato de metilo & $4 \mathrm{~mL} /$ bote/árbol \\
7 & Aspersión de Agua + leche nido + azúcar & $1 \mathrm{~L}+5 \mathrm{~g}+5 \mathrm{~g} /$ árbol \\
\hline
\end{tabular}


Cuadro 2. Suplementos alimenticios y atrayente evaluados para atraer y/o retener depredadores de escama blanca del mango Aulacaspis tubercularis. Sitio dos. Atonalisco, Nayairt. Ciclos 2009 y 2010.

\begin{tabular}{cll}
\hline Tratamiento & \multicolumn{1}{c}{ Descripción } & \multicolumn{1}{c}{ Dosis } \\
\hline 1 & Testigo absoluto & Sin aplicación \\
2 & Agua & $1 \mathrm{~L} /$ árbol \\
3 & Agua + azúcar estándar & $1 \mathrm{~L}+5 \mathrm{~g}$ \\
4 & Agua + leche nido & $1 \mathrm{~L}+5 \mathrm{~g}$ \\
5 & Agua + levadura de cerveza (Saccharomyces cerevisae) & $1 \mathrm{~L}+5 \mathrm{~g}$ \\
6 & Agua + leche nido+ azúcar & $1 \mathrm{~L}+5 \mathrm{~g}+5 \mathrm{~g}$ \\
7 & Agua + levadura de cerveza + azúcar & $1 \mathrm{~L}+5+5 \mathrm{~g}$ \\
\hline
\end{tabular}

colocó en una rama en el centro del árbol, a una altura aproximada de $2 \mathrm{~m}$. El SM se aplicó en una sola ocasión al inicio del estudio.

Sitio dos. La evaluación se realizó en un huerto ubicado en los terrenos del ejido Atonalisco, Tepic, Nayarit; cuyas coordenadas geográficas son $21^{\circ} 38^{\prime} 3.8^{\prime \prime} \mathrm{N}$ y $104^{\circ} 48^{\prime}$ 39.4" O, a una altura de 511 metros sobre el mar. La edad del huerto era de 13 años, plantado en marco real de $10 \times 10 \mathrm{~m}$. La humedad relativa y temperatura promedio durante las observaciones en los dos ciclos de evaluación fueron $59.95 \%$ y $23.21{ }^{\circ} \mathrm{C}$, $72.0 \%$ y $21.5^{\circ} \mathrm{C}$, en el primer y segundo ciclo, respectivamente. Las aplicaciones en la primera evaluación iniciaron el 10 de marzo y concluyeron el 28 de abril de 2009, en el segundo ciclo las aplicaciones iniciaron el 22 de enero y concluyeron el 13 de mayo de 2010. En total se efectuaron siete aplicaciones. Las aplicaciones de los suplementos alimenticios se realizaron con una bomba manual de mochila de $15 \mathrm{~L}$ de capacidad y 87 psi, se utilizó una boquilla de cono hueco. Se asperjó alrededor de la copa del árbol a una altura aproximada de dos metros, se utilizó agua como vehículo con un $\mathrm{pH}$ de 7.2, el gasto de la mezcla fue de un litro por árbol. Durante el periodo de observaciones en ambos sitios no se aplicaron insecticidas ni algún otro agroquímico que pudiera interferir con los resultados. Los tratamientos se enlistan en el cuadro 2.

Leche en polvo utilizada para el estudio. Se utilizó la marca comercial Nido Kinder $1^{+1}$ cuya composición es la siguiente: Proteínas mínimo $15.0 \mathrm{~g}$, lípidos $20.0 \mathrm{~g}$, carbohidratos $52.5 \mathrm{~g}$ de los cuales $8.6 \mathrm{~g}$ son de azúcares, fibra dietética $2.7 \mathrm{~g}$, Sodio $250 \mathrm{mg}$, Calcio $740 \mathrm{mg}$, Hierro $6.0 \mathrm{mg}$, Zinc $5.0 \mathrm{mg}$, Vitamina C $40 \mathrm{mg}$, Niacina 6.5 $\mathrm{mg}$, Vitamina E $5.0 \mathrm{mg}$, Acido Pantoténico $2.2 \mathrm{mg}$, Vitamina B6 $0.50 \mathrm{mg}$, B1 0.45 mg, Vitamina A eq. Retinol. $125 \mu \mathrm{g}$, Biotina $50 \mu \mathrm{g}$, Vitamina K $19.0 \mu \mathrm{g}$, Vitamina D $3.5 \mu \mathrm{g}$.

\footnotetext{
${ }^{1}$ Marca comercial propiedad de la empresa Nestlé.
} 


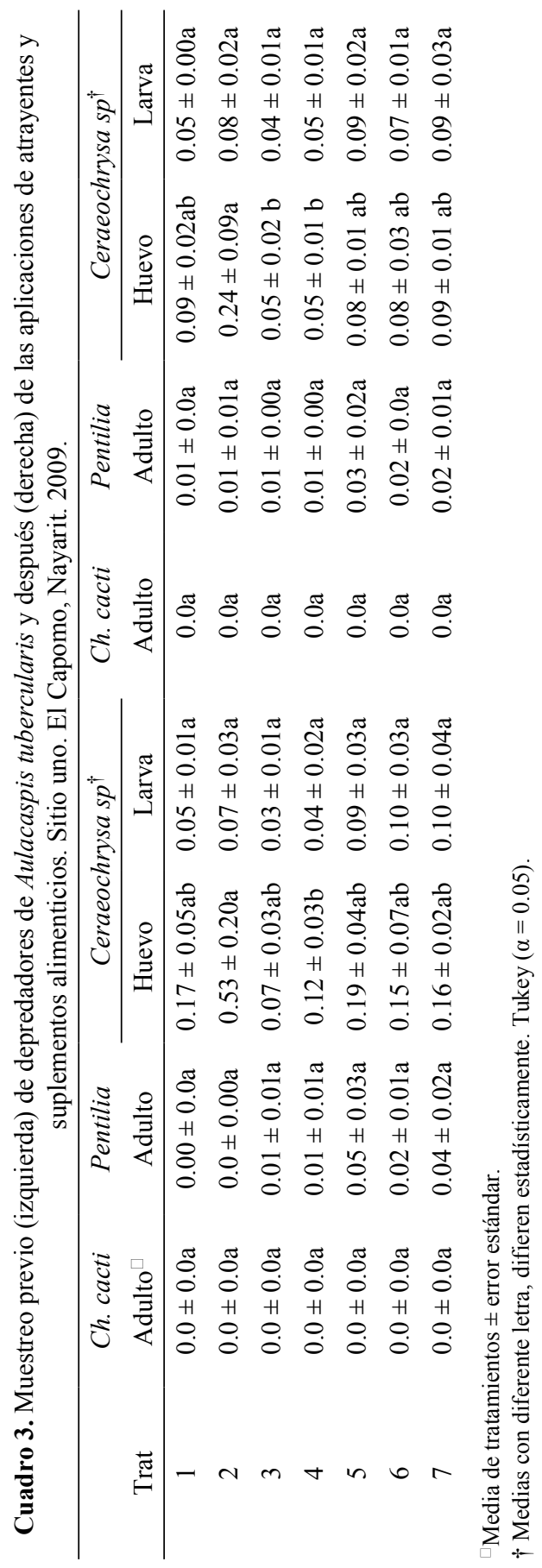


La levadura de cerveza (Sacharomyces cerevisae) utilizada en el estudio fue en su forma desamargada.

Los depredadores considerados en los dos sitios de estudio fueron: Ceraeochrysa sp, Chilochorus cacti L. y Pentilia sp

Las variables evaluadas fueron:

Número de huevos y larvas/brote (Ceraeochrysa sp), número de adultos y larvas/ brote (Chilochorus cacti), número de adultos/brote (Pentilia $\mathrm{sp)} \mathrm{y} \mathrm{número} \mathrm{de} \mathrm{colo-}$ nias de ninfas de EBM/hoja

Se seleccionó una rama de cada punto cardinal, en ésta se contabilizaron los individuos observados en el penúltimo y último brote vegetativo de acuerdo a lo sugerido por Urias et al. (2010). La variable ninfas por hoja se midió al inicio de cosecha, para ello se contó el total de colonias de ninfas vivas en dos hojas, una en el penúltimo y otra en el último brote vegetativo de cada punto cardinal del árbol. Se realizó análisis de varianzas para detectar diferencias entre tratamientos con $\alpha \leq 0.05$ y comparación de medias de tratamientos con la prueba de Tukey $(\alpha \leq 0.05)$ en las variables donde se observó diferencias significativas.

El diseño experimental fue en bloques completamente al azar con seis repeticiones, se utilizó un árbol como unidad experimental en ambas localidades. El análisis de varianzas y comparación de medias fue realizado con el promedio final. Se utilizó el programa estadístico SAS versión 9.2. Se realizaron muestreos previos a las aplicaciones de tratamientos para detectar diferencias significativas $(\alpha \leq 0.05)$ en los niveles poblacionales de los depredadores observados y evitar el efecto de covariables. Una covariable es aquella en la cual el investigador no tiene control pero puede estimar su efecto, se relaciona e influye en la respuesta de la variable de interés (Montgomery 2008).

\section{RESULTADOS Y DISCUSIÓN}

Sitio uno 2009. En los niveles de huevos por brote previos a las aplicaciones, se observaron diferencias significativas $(p \leq 0.05)$ entre tratamientos, por lo anterior en la comparación de las medias se incluyó el muestreo previo como covariable.

En este ciclo de evaluación para la variable huevos de Ceraeochrysa posterior a la aplicación se observaron diferencias significativas $(p \leq 0.05)$ entre tratamientos (Cuadro 3). El promedio más alto se observó para el tratamiento dos con la dosis más baja ( $0.25 \mathrm{~mL} /$ árbol) de SM con 0.24 huevos por brote, no obstante, si observamos el muestreo previo, los promedios posteriores, aunque con menor densidad, se mantienen similares; si bien se realizó el análisis de covarianza, es probable que las diferencias posteriores sean atribuibles a los diferentes niveles poblacionales iniciales. Los promedios más bajos se observaron en los tratamientos tres y cuatro (0.5 y 1.0 $\mathrm{mL} /$ árbol de SM, respectivamente) con 0.05 huevos/brote. 
Para el caso de la variable larvas de Ceraeochrysa, no se observaron diferencias significativas $(p>0.05)$; no obstante, numéricamente en los tratamientos uno, tres y cuatro se observó la menor cantidad de larvas. En el caso de Pentilia sp se observaron ligeras diferencias numéricas sin significancia estadística $(p>0.05)$. Chilocorus cacti no ocurrió en alguno de los tratamiento durante el periodo de observación. Situación similar observó Lee (2010) al aplicar SM en el cultivo de fresa para incrementar los enemigos naturales de pulgones y ácaros, no detectó diferencias significativas en la captura del depredador Pterostichus melanarius; no obstante menciona que ocurrieron ligeros incrementos de otros depredadores. Por su parte, Zhu \& Park (2005) observaron gran atracción de Coccinella septempunctata L. en trampas cebadas con SM, lo contrario ocurrió con Harmonia axyridis Pallas. De la misma manera, James (2003) al cebar trampa con SM, observó atracción de Chrysopa nigricornis Burmeister. Lo anterior indica diferente respuesta de atracción hacia SM con base en la especie de depredador observada.

En la variable número de colonias de ninfas por hoja (Cuadro 4), no hubo diferencias significativas entre tratamientos, sin embargo se pueden observar diferencias numéricas entre ellos, el promedio menor ocurrió con la aplicación del tratamiento seis (4 mL/brote/árbol de SM), el cual presentó 1.67 colonias/hoja, lo cual equivale a $60 \%$ menos de colonias que el testigo absoluto, es probable, que esta disminución se deba a la acción conjunta de todos los depredadores.

Sitio dos, año 2009. En el muestreo previo de huevos por brote, se observo diferencia significativa entre tratamientos. Por lo cual se realizó el análisis de covariable como ocurrió en el sitio uno.

En los muestreos posteriores a las aplicaciones, ocurrieron diferencias significativas $(p=0.01)$ en la variable huevos de Ceraeochrysa spp por brote (Cuadro 5),

Cuadro 4. Colonias de ninfas de Aulacaspis tubercularis.

En los tratamientos con atrayentes y suplementos alimenticios.

Sitio uno. El Capomo, Nayarit. 2009.

\begin{tabular}{ll}
\hline Tratamiento & Colonias de ninfas/hoja ${ }^{\square}$ \\
\hline Testigo absoluto & $4.17 \pm 1.30 \mathrm{a}$ \\
Salicilato de metilo & $1.75 \pm 0.63 \mathrm{a}$ \\
Salicilato de metilo & $2.25 \pm 0.76 \mathrm{a}$ \\
Salicilato de metilo & $2.00 \pm 0.63 \mathrm{a}$ \\
Salicilato de metilo & $2.67 \pm 0.68 \mathrm{a}$ \\
Salicilato de metilo & $1.67 \pm 0.42 \mathrm{a}$ \\
Aspersión de Agua + leche nido+ azúcar & $1.92 \pm 0.47 \mathrm{a}$ \\
\hline${ }^{\top}$ Media de tratamientos \pm error estándar. & \\
${ }^{\dagger}$ Medias con diferente letra, difieren estadísticamente. Tukey $(\alpha=0.05)$.
\end{tabular}




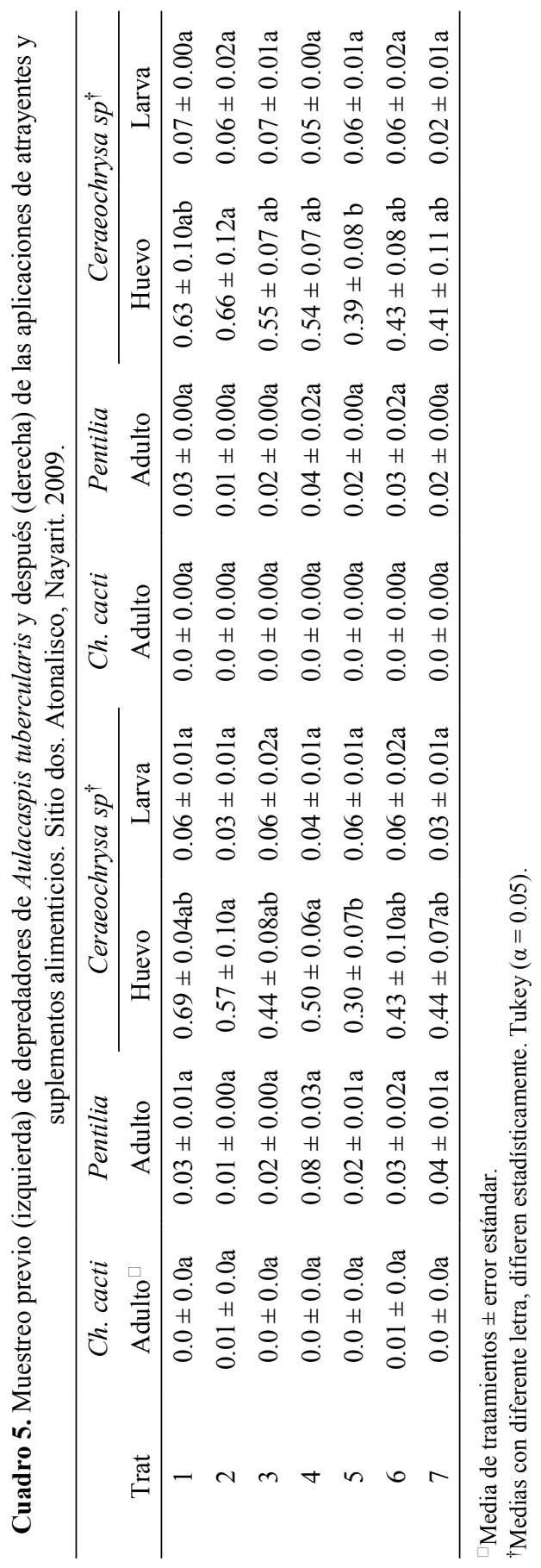


se observaron tres grupos estadísticos, en el tratamiento cinco ( $1 \mathrm{~L}$ de agua $+5 \mathrm{~g}$ de levadura de cerveza), se observó la menor cantidad de huevos, $38 \%$ menos que el testigo absoluto, por el contrario, en el tratamiento dos ( $1 \mathrm{~L}$ de agua) ocurrió la mayor cantidad de huevos.

En el número de larvas de Ceraeochrysa sp. no ocurrieron diferencias significativas entre los tratamientos, se observaron números similares al análisis previo, los tratamientos evaluados en este sitio no ejercieron algún efecto sobre esta variable. Situación análoga ocurrió con adultos de Pentilia y Chilocorus cacti. Se concluye que los tratamientos evaluados en las condiciones en que se desarrolló la presente investigación, no ejercieron un efecto significativo en el incremento de las variables observadas.

El número de colonias de escama por hoja fue diferente $(\mathrm{p} \leq 0.05)$ entre tratamientos (Cuadro 6), se observa el promedio menor en los tratamientos con base en agua más levadura de cerveza y azúcar ( $1 \mathrm{~L}+5 \mathrm{~g}+5 \mathrm{~g}$, respectivamente), agua más leche en polvo y azúcar ( $1 \mathrm{~L}+5 \mathrm{~g}+5 \mathrm{~g}$, respectivamente) y agua más levadura de cerveza ( $1 \mathrm{~L}+5 \mathrm{~g}$ ), se observa como factor común la levadura de cerveza, por lo que quizás ésta ejerza un estímulo en los depredadores para alimentarse de escama blanca. Por lo anterior, es recomendable evaluar tratamientos en estudios posteriores con diferentes concentraciones de levadura. Al ocurrir menor población de escama blanca en ambos sitios y escasas diferencias significativas en la población de depredadores, se concluye una acción conjunta de los depredadores para el control de EBM.

Sitio uno. 2010. En el muestreo previo no se observaron diferencias significativas ( $p$ $>0.05$ ) entre los tratamientos. Al observar los niveles poblaciones de cada variable posterior a las aplicaciones, no ocurren diferencias significativas entre los tratamientos, hay aumentos respecto a lo observado previamente, no obstante ocurrió lo mismo

Cuadro 6. Colonias de ninfas de Aulacaspis tubercularis en los tratamientos con atrayentes y suplementos alimenticios. Sitio dos Atonalisco, Nayarit. 2009.

\begin{tabular}{ll}
\hline Tratamiento & Colonias de ninfas/hoja ${ }^{\dagger}$ \\
\hline Testigo absoluto & $4.58 \pm 1.44 \mathrm{~b}$ \\
Agua & $4.00 \pm 0.53 \mathrm{bc}$ \\
Agua + azúcar estándar & $7.83 \pm 1.90 \mathrm{a}$ \\
Agua + leche nido & $5.16 \pm 1.21 \mathrm{ab}$ \\
Agua + levadura de cerveza (Saccharomyces cerevisae) & $2.91 \pm 0.88 \mathrm{bcd}$ \\
Agua + leche nido+ azúcar & $1.50 \pm 0.38 \mathrm{~cd}$ \\
Agua + levadura de cerveza + azúcar & $0.25 \pm 0.17 \mathrm{~d}$ \\
\hline
\end{tabular}

$\square$ Media de tratamientos \pm error estándar.

${ }^{\dagger}$ Medias con diferente letra, difieren estadísticamente. Tukey $(\alpha=0.05)$. 


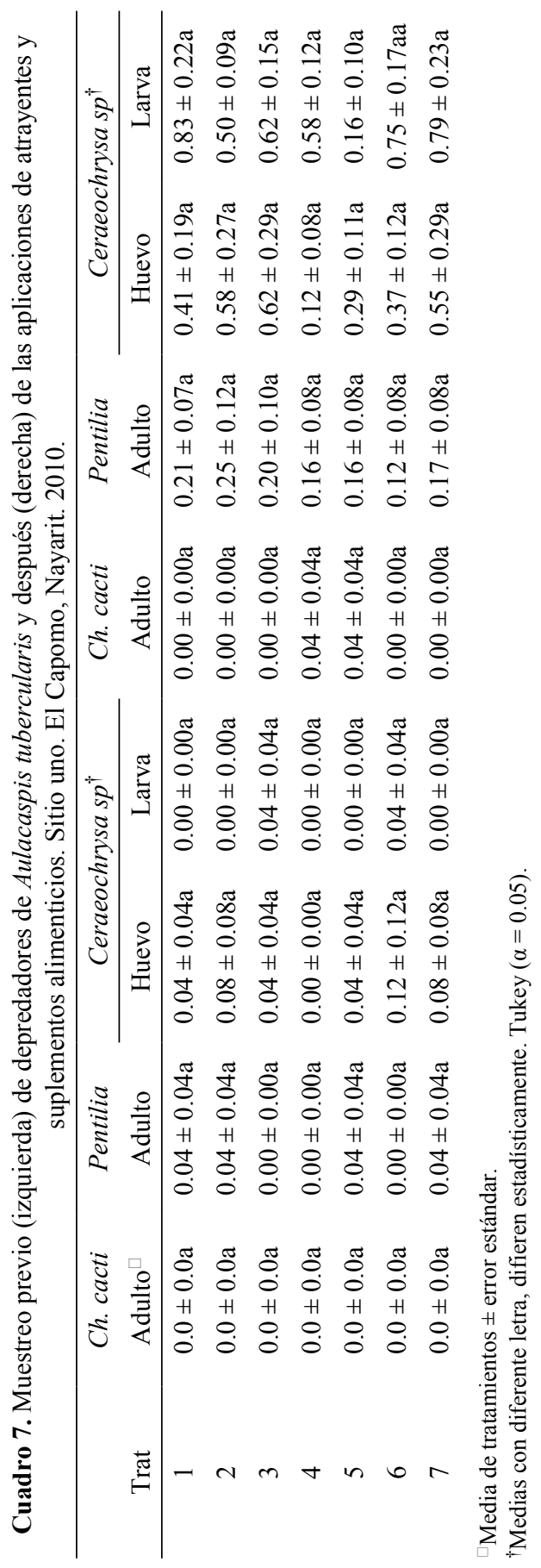


en el testigo absoluto (Cuadro 7). Al igual que el año anterior, excepto en los tratamientos cuatro y cinco ( 1 y $2 \mathrm{~mL}$ de $\mathrm{SM}$, respectivamente), no se observaron adultos de Chilocorus cacti.

En el número de colonias de ninfas por hoja (Cuadro 8), aunque sin diferencia significativa $(p>0.05)$, el promedio menor se observó en los tratamientos dos y siete ( $0.25 \mathrm{~mL}$ de SM y $1 \mathrm{~L}$ de agua $+5 \mathrm{~g}$ de azúcar $+5 \mathrm{~g}$ de leche en polvo), en éstos se observó $46.78 \%$ y $45.87 \%$, respectivamente, menos colonias de escamas por hoja que el testigo. El tratamiento $6(4 \mathrm{~mL}$ de $\mathrm{SM})$ presentó similar número de colonias de escamas que el testigo.

Sitio dos. 2010. El número de larvas y huevos de Ceraeochrysa al igual que adultos de Pentilia no mostró diferencia estadísticamente entre tratamientos (Cuadro 9). En este segundo año de evaluación, con excepción de los tratamientos 2 y 7 , se observaron adultos de Chilocorus cacti en los tratamientos evaluados.

En el número de colonias de ninfas por hoja (Cuadro 10), se observó el promedio menor en el testigo. Se encuentra un elemento común en los tratamientos dos, tres cuatro y seis los cuales presentan agua, azúcar y leche. Otro elemento común observado, fue la levadura presente en los tratamientos cinco y siete, lo cual coincide con lo observado en el experimento realizado en el 2009.

\section{CONCLUSIONES}

De acuerdo con el objetivo planteado, se puede concluir que los tratamientos con salicilato de metilo en el sitio uno (Las Varas, Nayarit, México) en ambos ciclos de evaluación, no mostraron diferencias significativas entre los tratamientos aplicados sobre el número de depredadores de escama blanca del mango. Se observó una diferencia

Cuadro 8. Colonias de ninfas de Aulacaspis tubercularis en los tratamientos con atrayentes y suplementos alimenticios. Sitio uno. El Capomo, Nayarit. 2010.

\begin{tabular}{lc}
\hline \multicolumn{1}{c}{ Tratamiento } & Colonias de ninfas $/ \mathrm{hoja}^{\square \dagger}$ \\
\hline Testigo absoluto & $1.09 \pm 0.15 \mathrm{a}$ \\
Salicilato de metilo & $0.58 \pm 0.20 \mathrm{a}$ \\
Salicilato de metilo & $0.87 \pm 0.22 \mathrm{a}$ \\
Salicilato de metilo & $0.66 \pm 0.40 \mathrm{a}$ \\
Salicilato de metilo & $0.79 \pm 0.20 \mathrm{a}$ \\
Salicilato de metilo & $1.03 \pm 0.25 \mathrm{a}$ \\
Aspersión de Agua + leche nido+ azúcar & $0.59 \pm 0.16 \mathrm{a}$ \\
\hline
\end{tabular}

$\square$ Media de tratamientos \pm error estándar.

${ }^{\dagger}$ Medias con diferente letra, difieren estadísticamente. Tukey $(\alpha=0.05)$. 


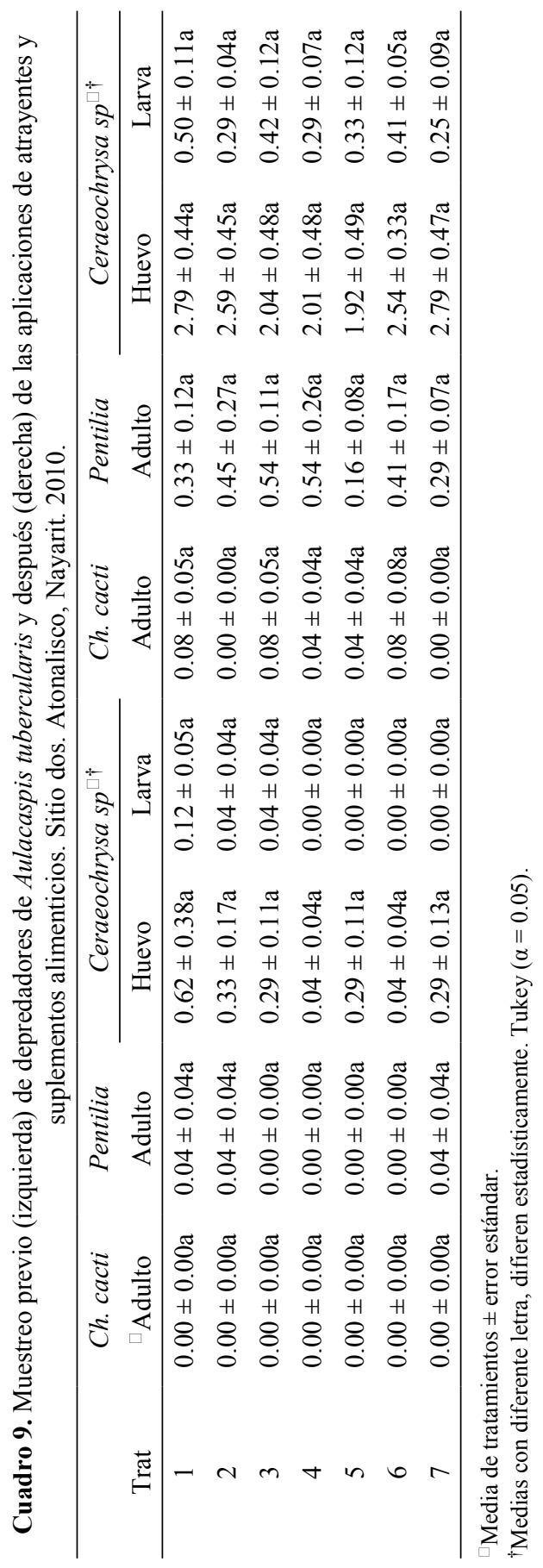


Hernández-Fuentes et al.: Atrayentes y suplementos para depredadores de Aulacaspis

Cuadro 10. Colonias de ninfas de Aulacaspis tubercularis en los tratamientos con atrayentes y suplementos alimenticios. Sitio dos. Atonalisco, Nayarit. 2010.

\begin{tabular}{lc}
\hline \multicolumn{1}{c}{ Tratamiento } & Colonias de ninfas/hoja $\square$ \\
\hline Testigo absoluto & $0.04 \pm 0.01 \mathrm{~b}$ \\
Agua & $0.26 \pm 0.08 \mathrm{ab}$ \\
Agua + azúcar estándar & $0.25 \pm 0.06 \mathrm{ab}$ \\
Agua + leche nido & $0.17 \pm 0.05 \mathrm{ab}$ \\
Agua + levadura de cerveza (Saccharomyces cerevisae) & $0.46 \pm 0.13 \mathrm{a}$ \\
Agua + leche nido+ azúcar & $0.20 \pm 0.07 \mathrm{ab}$ \\
Agua + levadura de cerveza + azúcar & $0.46 \pm 0.09 \mathrm{a}$ \\
\hline
\end{tabular}

$\square$ Media de tratamientos \pm error estándar.

${ }^{\dagger}$ Medias con diferente letra, difieren estadísticamente. Tukey $(\alpha=0.05)$

numérica en cuanto a huevos y larvas de Ceraeochrysa sp las cuales se presentaron en mayor proporción durante el estudio. Al respecto, De Boer \& Dicke (2004), al evaluar diferentes dosis de SM para atraer a Phytoseiulus persimilis, observaron una respuesta dosis-dependiente. Nuestros resultados y los obtenidos en estudios de campo con el uso de SM sintético realizados por James \& Price (2004), Zhu \& Park (2005) y Lee (2010) para atraer y/o retener depredadores podrían indicar especificidad de atracción. Para el sitio dos (Atonalisco, Nayarit), con la aplicación de suplementos alimenticios con base en agua + levadura de cerveza y azúcar, se observó una diferencia numérica importante de huevos de Ceraeochrysa sp; en ambos sitios de estudio la presencia de Chilocorus cacti fue muy baja, en algunos casos su valor numérico fue cero, concluyéndose que los tratamientos evaluados no ejercieron ningún efecto significativo sobre éste. Pentilia sp. se presentó en mayor cantidad en ambos sitios, con un ligero aumento en el sitio dos (Atonalisco). La disminución de colonias de escama podría ser el resultado de un incremento general de los depredadores observados y la acción conjunta de éstos. Es conveniente pues, realizar mayores observaciones al respecto incluyendo nuevas dosis de los tratamientos evaluados.

Agradecimientos. Las actividades realizadas en esta investigación fueron financiadas por el fondo Sectorial SAGARPA-CONACYT mediante el proyecto 48122/2006. Se agradece la disposición de los agricultores para apoyar con los huertos donde se realizaron las actividades.

\section{LITERATURA CITADA}

Barbosa, P. 1998. Conservation Biological Control. Academic Press. San Diego. 396 pp.

Cardinale, B. J., C. T. Harvey, K. Gross \& A. R. Ives. 2003. Biodiversity and biocontrol: Emergent impacts of a multi-enemy assemblage on pest suppression and crop yield in an agroecosystem. Ecology Letters, 6: 857-865. 
Croft, B. A. 1990. Arthropod Biological Control Agents and Pesticides. John Wiley and Sons, New York. 723 pp.

De Boer, J. \& M. Dicke. 2004. The role of methyl salicilate in prey searching behavior of the predatory mite Phytoseiulus persimilis. Journal of Chemical Ecology, 30: 255-271.

Dethier, A. 1947. Chemical insect attractans and repellents. The Maple Press Company, E.U. 289 pp.

Dicke, M., T. A. Beek, M. A. Posthumus, N. Ben Dom, V. H. Bokhoven \& A. E. Groot. 1990. Isolation and identification of volatile kairomone that affects acarine predator-prey interactions. Involvement of host plant in its production. Journal of Chemical Ecology, 16: 381-396.

Dicke, M., R. Gols, D. Ludeking \& M. A. Posthumus. 1999. Jasmonic acid and herbivory differentially induce carnivore-attracting plant volatiles in lima bean plants. Journal of Chemical Ecology, 25: 1907-1922.

FAOSTAT. 2007. Food and Agriculture Organization of the United Nations. En línea: http://www.fao. org/economic/ess/ess-publications/ess-yearbook/anuario-estadistico-de-la-fao-2007-2008/a-recursos/es/ (consultado el 09 de diciembre de 2010).

Galán, S. V. 1999. El cultivo del mango. Ed. Mundi-Prensa. Madrid, España. 298 pp.

García A. N. C., M. A. Urias L., J. A. González C., L. M. Hernández F., V. Vázquez V. \& H. Pérez B. 2009. Productos novedosos para el control de la escama blanca del mango, Aulacaspis tubercularis Newstead (Hemíptera: Diaspididae). Entomología Mexicana, 8: 642-647.

Gonzalez, C. J. A., M. A. Urias L., L. M. Hernandez F., J. G. Lopez A. \& N. C. García A. 2010. Poblaciones de especies de cargabasura (Neuroptera: Chrysopide) asociadas a la escama blanca del mango en Nayarit. pp. 288-291. In: Memoria del XXXIII Congreso Nacional de Control Biológico. Uruapan, Michoacán, México.

Hegde, M., J. N. Oliveira, J. G. da Costa, E. Bleicher, A. E. G. Santana, T. J. A. Bruce. J. Caulfield, S. Y. Dewhirst, C. M. Woodcock, J. A. Pickett \& M. A. Birkett. 2011. Identification of semiochemicals released by cotton Gossypium hirsutum, upon infestation by the cotton aphid, Aphis gossypii. Journal of Chemical Ecology, 37: 741-750.

James, G. D., S. C. Castle \& T. V. Grasswitz. 2005. Using synthetic herbivor-induced plant volatiles to enhance conservation biological control: field experiments in hops and grapes. pp 192-205. In: Second International Symposium on Biological Control of Arthropods. Davos, Suiza.

James, D. G. \& T. S. Price. 2004. Field-testing of methyl salicylate for recruitment and retention of beneficial insects in grapes and hops. Journal of Chemical Ecology, 30: 1613-1628.

James, D. G. 2003. Synthetic herbivore-induced plant volatiles as attractants for beneficial insects. Environmental Entomology, 32: 977-982.

Jervis, M. A. \& G. E. Heimpel. 2007. Phytophagy, pp. 526-550. In: Jervis M. A. (Ed.). Insects as natural enemies: a practical perspective. Ed. Springer. Dordrecht, The Netherlands. 748 pp.

Jepson, P. C. (Ed.). 1989. Pesticides and Non-Target Invertebrates. Intercept, Wimborne, Dorset, United Kingdom. 240 pp.

Kfir, R. \& D. Rosen. 1980. Parasites of soft scale (Homoptera:Coccidae) in Israel; an annotated list Journal of the Entomological Society of South Africa, 43: 113-128.

Labuschagne, T. I. 1993. Progress with integrated control of the mango scale Aulacaspis tubercularis Newstead. South Africa Mango Growers Association Yearbook 13: 134-135.

Lee, C. Y. 2010. Effect of methyl salicylate-based lures on beneficial and pest arthropods in strawberry. Environmental Entomology, 39: 653-660.

Montgomery, D. C. 2008. Diseño y análisis de experimentos. 2da. Edición. Ed. Limusa. México. 686 pp.

Peña, J. E. \& A. I. Mohyuddin. 1997. Insect pest, pp 327-362. In: R. E. Litz (Ed.). The mango: botany, production and uses. CAB International. $587 \mathrm{pp}$. 
SIAP-SAGARPA. 2009. Anuario estadístico de la producción agropecuaria. SIAP-SAGARPA. México. En línea: http://www.siap.gob.mx/index.php?option=com_wrapper\&view=wrapper\&Itemid=35 1. Consultado el 25 de octubre de 2010.

Stehr, W. F. 1990. Parásitos y depredadores en el manejo de plagas, pp. 173-221. In: R. L. Metcalf \& W. H. Luckmann (Eds.). Introducción al manejo de plagas de insectos. Ed. Limusa. México. 710 pp.

Urías, L. M. A. 2006. Principales plagas del mango en Nayarit, pp. 211-234. In: V. Vázquez V. \& M. H. Pérez B. (Eds.). El cultivo del mango: Principios y tecnología de producción. INIFAP. México. $321 \mathrm{pp}$.

Urías, L. M. A., J. A. Osuna G, V. Vázquez \& M. H. Pérez B. 2010. Fluctuación poblacional y distribución de la escama blanca del mango (Aulacaspis tubercularis Newstead) en Nayarit, México. Revista Chapingo Serie Horticultura, 16: 77-82.

Urías, L. M. A. \& R. Flores C. 2005. La escama blanca, Aulacaspis tubercularis Newstead (Homoptera: Diaspididae) una nueva plaga del mango: fluctuación poblacional y anotaciones biológicas. Entomología Mexicana, 4: 579-584.

Van Driesche, R. G. \& T. S. Bellows. 1996. Biological control. Chapman and Hall. New York, E.U.A. $539 \mathrm{pp}$.

Waage, J. K. \& D. Greathead (Eds.). 1986. Insect Parasitoids. Academic Press, London. U. K. 389 pp.

Wysoki, M., Y. Ben-Dov E. Swirski \& Y. Izhar. 1993. The arthropod pests of mango in Israel. Acta Horticulturae 341: 452-466.

Zhu, J. \& K. C. Park. 2005. Methyl salicylate, a soybean aphid-induced planta volatile attractive to the predator Coccinella septempunctata. Journal of Chemical Ecology, 31: 1733-1746. 